\title{
ATOM IN A STRONG MAGNETIC FIELD. TRANSFORMATION OF ATOMS INTO TRANSATOMS
}

\section{Gennady V. Mishinsky}

Joint Institute for Nuclear Research, http://www.jinr.ru

Dubna, Moscow Region 141980, Russian Federation

mysh@jinr.ru

Abstract. All $\ell$-s and $\mathbf{j}-\mathbf{j}$ bonds in a strong magnetic field $\mathbf{B}$ are broken in all atomic electrons and their orbital moments are "frozen-in" in the field. Electronic states with identical orbital $\ell$ and magnetic moments $m_{\ell}$ are split into two levels with antiparallel electron spins $s= \pm 1 / 2$. The frequency of transitions between these levels $m_{s}= \pm 1 / 2$ is the same for all electron pairs $\omega=2 \mu_{\mathrm{e}} \mathrm{B} / \hbar$. Electromagnetic interaction of electrons causes them to oscillate near their orbitals. These oscillations are quantized by introducing a new quantum number. The exchange interaction between two electrons, an additional asymmetry in their oscillations enable the electrons to create an ortho-boson with $S=1$. The orbital magnetic moment $m_{\ell}$ of each electron precesses around the magnetic field $B$ with a frequency $\omega=\ell \cdot \mu_{e} B / \hbar$ and creates its own internal magnetic field $B_{\mu}$ rotating with the same frequency. The internal magnetic field $B_{\mu}$ stimulates transitions between the levels $m_{s}=1 / 2 \rightarrow m_{s}=-1 / 2$. Thus, ortho-bosons are formed in the entire atom and electron Bose-Einstein condensate is produced. Consequently, atoms, in a strong magnetic field, inevitably turn into transatoms.

Keywords: atomic physics, quantum physics, electron Bose-Einstein condensate, the Pauli principle, electron magnetic resonance, coherent radiation, condensed matter nuclear science

PACS: 36.10.-k; 32.10.-f; 76.30.-v; 03.75.Mn; 03.75.Nt; 42.55.Vc

Bibliography - 27 references

Received 14.11.2017

RENSIT, 2017, 9(2):147-160

DOI: $10.17725 /$ rensit.2017.09.147

\section{ConTENTS}

1. INTRODUCTION (147)

2. Atom in weak, medium and ultrastrong MAGNETIC FIELDS (148)

3. Аtom in strong magnetic fields. Electron oscillations (149)

4. Electron PAIRING INTO ORTHO-BOSON (152)

5. INTRA-ATOMIC ELECTRON MAGNETIC RESONANCE (154)

6. Атом as Z/2 active medium (156)

7. Formation OF TRANSATOMS TRANSMOLECULES (157)

8. Conclusion (158)

REFERENCES (159)

\section{INTRODUCTION}

"The electron is as inexhaustible as the atom, nature is infinite, but it exists infinitely ..." [1]. This statement is associated with the development, in the early 20th century, of the theory of

AND knowledge. The author of this statement did not, at the time of writing that article, yet know that electrons are part of atoms, and he considered them to be independent substances. For physicists, this phrase should sound the opposite: the atom is inexhaustible like the electron. Such a turn is caused, in the theory of systems, by the dependence of properties of the system on the state of its constituent elements, and on the changing behavior of their existence in changing external conditions. In our case, the atom is a system, and the electrons, along with atomic nucleus, are its constituent elements. Changes in properties and behavior, both of electrons and nuclei, cause appropriate transformations in the properties of the atom. In this paper, we consider a change in atomic structure when an atom is placed in a strong magnetic field. In science and technology, magnetic fields are subdivided into weak - up to $0.05 \mathrm{~T}$, medium $-0.05 \div 4 \mathrm{~T}$; strong 
- 4:100T and ultrastrong fields - more than 100T. Furthermore, the presentation will adhere to that classification.

\section{ATOM IN WEAK, MEDIUM AND ULTRASTRONG MAGNETIC FIELDS}

The fine structure of optical spectra of atoms is explained by spin-orbit interaction, i.e. by the interaction of spin magnetic moments of all external electrons with magnetic field $B_{\mathrm{L}}$ produced by orbital motion of electrons in the atom. Calculations show that magnetic induction vector of the field created by orbital motion of electrons at the location of nucleus is $10 \div 200 T$ (for $\mathrm{Li} \sim 13 \mathrm{~T}$, for Cs $\sim 210 \mathrm{~T}$ ). States with different orientations of the total electron spin $\mathrm{S}$ and the associated magnetic moment $\mu_{\mathrm{S}}$ relative to $B_{\mathrm{L}}$ differ in energy by $\Delta E=-\mu_{\mathrm{S}} B_{\mathrm{L}}$, which causes a thin splitting of levels. The number of orientations $2 S+1$ is determined by total spin of electrons $S$. The simplest picture of interaction is obtained for atoms with one external electron. In this case, there are only two orientations of the magnetic moment of the electron $\mu_{\mathrm{e}}$ relative to $B_{\mathrm{L}}$ either along the field or against the field. Accordingly, two close lines are observed in the spectrum $\left(\mu_{\mathrm{e}}=e \hbar / 2 m e=9.29 \cdot 10^{-24} \mathrm{~J} / \mathrm{T}=5.79 \cdot 10^{-5} \mathrm{eV} / \mathrm{T}\right.$, where $\hbar$ is the Planck constant, $e$ and $m_{\mathrm{e}}$ are electron charge and mass).

Atoms with several electrons in the outer shell have a more complicated fine structure. Thus helium with two electrons has single lines in case of antiparallel orientation of electron spins with total zero spin $S=0$ - parahelium and triple lines in case of parallel orientation of electron spins with total spin $S=1-$ orthohelium. The spin $S=1$ corresponds to three possible projections on magnetic field direction: $+1,0,-1$.

The hyperfine structure of spectral lines is explained by magnetic field interaction $B_{\mathrm{L}}$ with magnetic moment of the nucleus $\mu_{\mathrm{I}}$ associated with its spin $I$. The extraordinary smallness of the hyperfine splitting is due to the smallness of nuclear magneton $\mu_{\mathrm{N}}=e h / 2 \mathrm{mP}=3.15 \cdot 10^{-8} \mathrm{eV} / \mathrm{T}$, where $m_{\mathrm{p}}$ is proton mass. When an atom is placed in an external magnetic field $\mathbf{B}$, the magnetic moments of electrons $\mu_{\mathrm{s}}$ and the magnetic moment of the nucleus $\mu_{\mathrm{I}}$ interact with both $\mathbf{B}$ and the magnetic field produced by the orbital motion of electrons in the $\mathbf{B}_{\mathrm{L}}$ atom. The energy of such interaction is $\Delta E_{\mathrm{e}}=-\mu_{\mathrm{S}}\left(B_{\mathrm{L}}+B\right), \Delta E_{\mathrm{I}}=-\mu_{\mathrm{I}}\left(B_{\mathrm{L}}+B\right)$.

Depending on influence on the structure of atomic levels, several cases of different values of $\mathbf{B}$ are distinguished. A weak external field $\mathbf{B}$ is a field that does not break the bonds between the nucleus $(I)$ and the electrons $(F=J+I)$ and between the orbital $(L)$ and the spin $(S)$ moments of the atom $(J=L+S)$ (Zeeman effect).

In the external field $\mathbf{B}$ of a medium value, first, the $I-J$ bond is broken and, secondly, the bond between the orbital and spin moments of the atom is broken: the L-S bond (the PaschenBuck effect). Historically, in physical literature, such a field is called a "strong" field. The Paschen-Buck effect is manifested in magnetic fields up to $4 T$, i.e. in medium fields, according to classification. Fig. 1 shows, as an example, the splitting in the weak (Fig. 1a) and medium magnetic fields of the ${ }^{2} S$ and ${ }^{2} P$ levels of the sodium atom. In case of the medium field (Fig. $1 \mathrm{~b})$, due to discontinuity of the $L-S$ bond, one can no longer refer to the total atomic moment of the $J$ atom. The ${ }^{2} P_{1 / 2}$ and ${ }^{2} P_{3 / 2}$ levels do not differ and are characterized as levels with $L=1$. The orbital moment of an atom in this case can
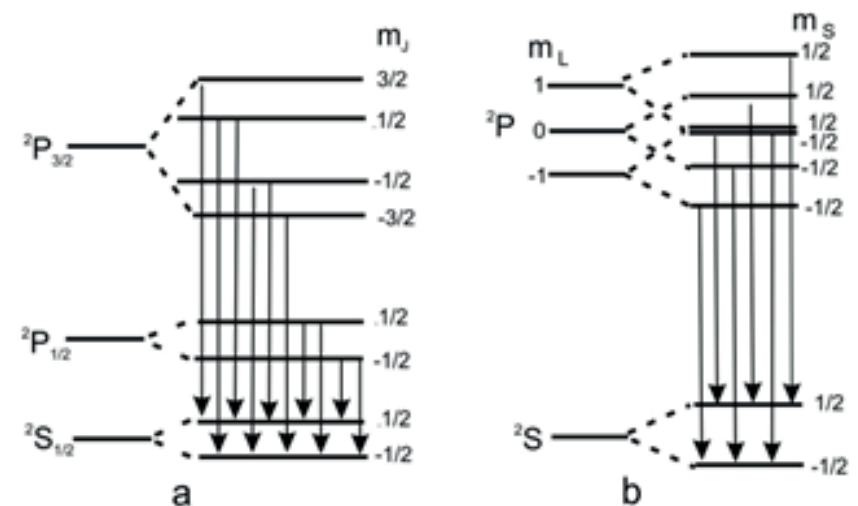

Fig. 1. Splitting of ground and excited energy levels of sodium atom: $a$ - in a weak external magnetic field and $b-$ in a medium magnetic field. 
be oriented relative to field $\mathbf{B}$ in three ways: $m_{\mathrm{L}}$ $=-1,0,1$. For each orientation of the orbital moment, the spin magnetic moment of an electron can be oriented in two ways, $m_{\mathrm{S}}= \pm 1 / 2$, for both $L=1$ and $L=0$. Electron levels with higher energy correspond to magnetic moments directed against the vector of magnetic induction. The projections of magnetic moments on the direction of magnetic field do not change at that. The condition for breaking the L-S bond occurs when the interaction energy of magnetic moment of an atom with an external magnetic field becomes greater than the energy of the spin-orbit interaction. In this case, the spin magnetic moment of the nucleus, the spin and orbital magnetic moments of the atom begin to interact independently with external magnetic field, increasing or decreasing the energy of an atom, depending on the orientation of magnetic moments with respect to the direction of magnetic induction vector $\mathbf{B}: \Delta E=-\mu_{\mathrm{I}} B-\mu_{\mathrm{L}} B-$ $2 \mu_{\mathrm{e}} S \cdot B$, where $\mu_{\mathrm{L}}=L \cdot \mu_{B}, \mu_{\mathrm{B}}=\mu_{\mathrm{e}}=e \hbar / 2 m_{\mathrm{e}}$. Each magnetic moment $\mu$ precesses independently the direction of magnetic induction vector of the external field with its frequency $\omega=\mu_{B} / \hbar$.

B.B. Kadomtsev showed in his papers [2$3]$ that in a heavy atom with a nuclear charge $Z>>1$ located in a ultrastrong magnetic field $\mathbf{B}$, with which the magnetic interaction of atomic electrons becomes larger than their Coulomb interaction and whose value exceeds significantly $10^{6} \mathrm{~T}\left(\mathrm{~B}>>10^{5} \cdot Z^{4 / 3} \mathrm{~T}\right)$, a complete rearrangement of electron configurations of all atomic electrons occurs. In this case, the electrons move in thin cylindrical shells, oscillating parallel to the axis of the magnetic field, and simultaneously rotate around the nucleus. At $B<<10^{5} \cdot Z^{3} T$, several electrons are located on each shell. In this case, the atom retains spherical symmetry, and its radius changes as $\left(Z / B^{2}\right)^{1 / 5}$, i.e. the atom contracts with increasing $\mathbf{B}$. For $B>>10^{5} \cdot Z^{4} T$, each individual electron precesses around the nucleus and makes motion in a thin cylindrical shell with a radius $\mathrm{R}$, oscillating along the magnetic field axis. The wave functions of individual electrons do not overlap, i.e. the exchange interaction is absent.

Thus, the structure of atomic levels varies both in the medium and ultrastrong magnetic fields. The question arises: How will change the structure of an atom in a strong magnetic field exceeding $20 T$ ?

\section{ATOM IN STRONG MAGNETIC FIELD. ELECTRON OSCILLATIONS}

It has been known that the state of an electron in an atom can be characterized by four quantum numbers: ground quantum number $n=1,2,3$, ...; orbital quantum number $\ell=0,1,2, \ldots, n-1$; magnetic quantum number $m_{\ell}=-\ell,-\ell+1, \ldots, 0, \ldots$, $\ell+1, \ell$; spin magnetic moment of an electron $m_{\mathrm{S}}=+1 / 2$ и $m_{\mathrm{S}}=-1 / 2$. According to Pauli principle, an atom cannot contain electrons with the same set of quantum numbers. Electrons with the same ground quantum number $\mathrm{n}$ form the shell of an atom. Fig. 2a shows the filling of shells by electrons in a sodium atom.

For clearness, the Bohr concept of elliptic motion of electrons in a plane will be used in reasoning and drawings.

The internal electron orbitals of the atom, in the absence of a strong magnetic field, do not have a constant orientation in outer space, since they precess under the action of other charges

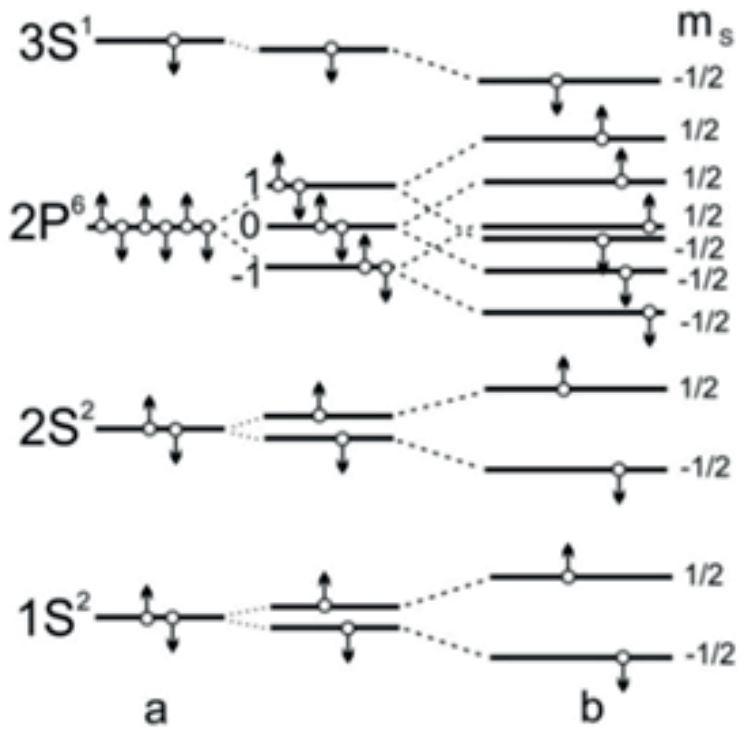

Fig. 2. $a$ - filling with electrons of sodium atom shell: $b-$ splitting of sodium atom energy levels in a strong magnetic field. 
and cease to lie in one plane. The electron moves around the nucleus not in a plane, but along a trajectory similar to "thread in the clew" [4]. Therefore, in spite of the fact that the orbital moments for $p, d \ldots$ of electronic states differ from zero $(\ell \neq 0)$, the medium values of orbital moments for $x$-, $y$ - and $z_{-}$-components are equal to zero: $\left(0\left|\ell_{\mathrm{x}}\right| 0\right)=\left(0\left|\ell_{\mathrm{y}}\right| 0\right)=\left(0\left|\ell_{\mathrm{z}}\right| 0\right)=0$. This phenomenon has received the name quenching orbital motion. Because of this, first, the magnetic field created by orbital motion of an electron is zeroed, $B=\mu_{0} \frac{e}{m_{c} c} \frac{l}{r^{3}}$, and, secondly, the forces of Coulomb repulsion between atomic electrons do not have a preferred direction.

In a strong external magnetic field, apparently, more than $20 \mathrm{~T}, \boldsymbol{\ell}-\boldsymbol{s}$ and $j-j$ bonds of each electron in the whole atom are torn apart, not only on external but also on internal orbitals. All electronic states are relieved from degeneration. The spin and orbital moments of each individual electron interact independently with an external magnetic field $\mathbf{B}$. The external magnetic field $\mathbf{B}$, which has a constant orientation, rigidly aligns electron orbitals with respect to its direction in accordance with their orbital magnetic moments. The average values of the orbital moments for $x-, y$ - and $z$-components cease to be zero. Orbital moments of electrons are "frozen-in" in a magnetic field. The atom in "amorphous state" is transformed into an ordered, magnetic "crystal". (The situation, in which magnetic moments of nanoclusters, which interact with a constant external magnetic field, are oriented along the field, is called magnetism of "frozenin" moments.)

In accordance with the orientation, the orbital moment $\ell$ of each electron and its associated orbital magnetic moment $\mu_{\ell}$ precess independently around the vector direction of external magnetic field with a frequency $\omega_{\ell}=\mu_{\ell} \cdot B / \hbar, \mu_{\ell}=\mu_{e} \cdot \ell$. Electron orbitals with equal orbital moment $\ell$ precess around $\mathbf{B}$ synchronously, with the same frequency $\omega_{\ell}$ (Fig. 3a). The spin magnetic moments of electrons do not precess around $\mathbf{B}$, since they are oriented only in two ways: $m_{\mathrm{S}}=-1 / 2$ over the field and $m_{\mathrm{S}}=+1 / 2$ against the field. The interaction energy of the orbital and spin magnetic moments of an electron is $\Delta \mathrm{E}=-\mu_{\mathrm{e}} \cdot \ell \cdot B-2 \mu_{\mathrm{e}} \cdot s \cdot B$. Fig. $2 \mathrm{~b}$ shows the splitting of energy, electronic levels in the sodium atom.

Electron orbitals align relative each other so that Coulomb repulsion forces between electrons are minimal. Fig. 3a shows schematically two orbitals with equal orbital moments $\ell$ and their projections $m_{\ell}$ on the $Z$ axis parallel to $\mathbf{B}$, but with different directions of electron spins $s= \pm 1 / 2$.

The Coulomb repulsive forces, which forced, in the absence of field $\mathbf{B}$, the electron orbitals to freely precess, now cause the electrons to oscillate near the orbitals. If atomic electrons oscillate, in case of an ultrastrong magnetic field [2], parallel to magnetic field axis at the expense of its interaction with magnetic moments of electrons, then in case of a strong magnetic field, the atomic electrons oscillate due to Coulomb repulsive forces between electrons and compensating Coulomb attraction forces of electrons to a positively charged nucleus.

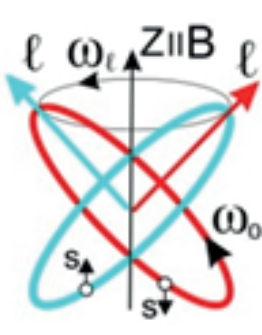

a

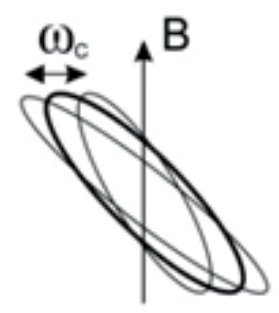

b

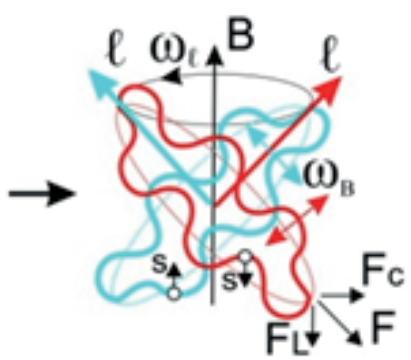

d

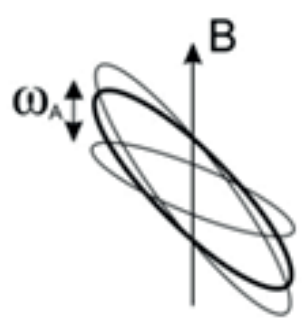

C
Fig. 3. $a-$ precession of two orbitals in magnetic field $\mathbf{B}$ with equal $\ell$ and $m_{\ell}$, but with different $s= \pm 1 / 2 ; b$ and $c$-electron oscillations transversely and along magnetic field direction; $d$ - electron oscillations close to orbitals in a strong magnetic field. 
The Coulomb repulsion force $F$ between electrons, which are located in adjacent orbitals with the same orbital magnetic moment, has a preferential direction and it can be decomposed into force components: one force $F_{c}$ acts transversely to the direction of magnetic induction vector and the second force $F_{\mathrm{L}}$ acts along the direction of magnetic induction vector (Fig. 3d). Transverse oscillations of $\omega_{C}$ can be represented as oscillations of entire electron orbital across the magnetic field near the equilibrium state (Fig. 3b). Longitudinal oscillations of $\omega_{\mathrm{A}}$ can be represented as oscillations that stretch and compress the orbital along magnetic field direction, near the same equilibrium state (Fig. 3c). Since transverse and longitudinal forces are components of one, total force, oscillation frequencies in transverse and longitudinal directions will be the same $\omega_{\mathrm{C}}=\omega_{\mathrm{A}} \equiv \omega_{\mathrm{B}}$ (Fig. 3d). However, oscillation amplitudes can be different. The oscillation frequency $\omega_{B}$ is related to electron rotation frequency on the orbital $\omega_{0}=E_{0} / \hbar$ by the following relation [5-6]

$$
\omega_{B}=k \sqrt{\omega_{0}^{2}+\omega_{l}^{2}} \text {, where } k=1,2,3 \ldots
$$

Since the rotational frequencies on the orbitals for electrons with different spins are not equal to each other: $\omega_{0}(s=1 / 2) \neq \omega_{0}(s=-1 / 2)$, then the oscillation frequencies for them will differ $\omega_{\mathrm{B}}(s=1 / 2) \neq \omega_{\mathrm{B}}(s=-1 / 2)$ even for equal numbers $k$ (1). However, since the energy of level $E_{0}$ is much greater than the energy of its splitting due to the interaction of field $\mathbf{B}$ both with orbital $\Delta E_{\ell}=\mu_{e} \cdot \ell \cdot B$, and spin $\Delta E_{\mathrm{s}}=2 \mu_{\mathrm{e}} \cdot s \cdot B$ electron magnetic moments:

$$
\begin{aligned}
& E_{0}>\Delta E_{\ell} \text { or } \omega_{0}>>\omega_{\ell}, \\
& E_{0}>\Delta E_{S} \text { or } \omega_{0}>>\omega_{s},
\end{aligned}
$$

where $\omega_{\mathrm{S}}=\Delta E_{\mathrm{S}} / \hbar$, then at equal numbers $k$, it follows from expression (2) that $\omega_{\mathrm{B}}(s=1 / 2) \approx$ $\omega_{0}(s=1 / 2)$ and $\omega_{\mathrm{B}}(s=-1 / 2) \approx \omega_{0}(s=-1 / 2)$, and it follows from expression (3) that

$$
\omega_{\mathrm{B}}(s=1 / 2) \approx \omega_{\mathrm{B}}(s=-1 / 2) \text {. }
$$

For orbitals with equal ground quantum number, orbital and magnetic quantum numbers, but with different electron spins, the number $k$ will be equal. This is due to the fact that electron energies on the orbitals are close to each other, the electrons are in one magnetic field and, in addition, synchronized electron oscillations minimize Coulomb repulsion between them.

Thus, atomic electrons in a strong magnetic field $\mathbf{B}$ not only rotate in orbitals that are strictly oriented and precess around the direction of the magnetic field $\mathbf{B}$, but the electrons additionally perform oscillations near their orbitals. Consequently, an additional degree of electron freedom appears in an atom in a strong magnetic field. A new degree of electron movement freedom generates a new quantum number for them.

It is known that a system with $j$ degrees of freedom is described by means of generalized coordinates $q_{\mathrm{i}}$ and generalized pulses $p_{\mathrm{i}}(i=1,2$, $3, \ldots, j)$. Each generalized moment pi depends on the corresponding generalized coordinate $q_{\mathrm{i}}$ only. The $j$ quantum conditions are superimposed on the motion of a system with $j$ degrees of freedom with $j$ quantum numbers $n_{\mathrm{i}}(i=1,2,3, \ldots, j)$ : $\oint p_{i} d q_{i}=2 \pi \hbar n_{i}\left(n_{\mathrm{i}}=1,2,3, \ldots ; i=1,2,3, \ldots, j\right)$

Thus, an intrinsic quantum condition is superimposed on the electron motion oscillating close to orbital, which can be correlated with the number $k$ from equation (1). Recall that in case of plane motion of an electron along an elliptical orbit around the nucleus, the system has two degrees of freedom [7]. Therefore, two quantum conditions are superimposed on this system expressed in two quantum numbers: azimuthal $n_{\varphi}$ and radial $n_{\mathrm{r}}$. The energies of electron stationary states in case of elliptical orbitals are characterized by the sum of the azimuthal and radial quantum numbers $n_{\varphi}+n_{\mathrm{r}}=n$ and they are equal to the energies of electron states in case of its motion along circular orbitals with the same values of ground quantum number $n$.

An atomic electron that moves in a strong magnetic field has three degrees of freedom. Consequently, it has an additional quantum number $n_{\mathrm{b}}$. The quantum number $n_{\mathrm{b}}$ allows that 
two or more electrons with different $n_{\mathrm{b}}$ have identically directed spins.

\section{ELECTRON PAIRING INTO ORTHO- BOSON}

If we transfer the conditions for electron pairing at superconductivity in metals to an atom, then we must observe three basic requirements:

1. The Pauli principle must be fulfilled, according to which fermions cannot be in exactly equal states;

2. Electrons should be attracted by each other (Cooper condition [8]). This attraction between electrons can be arbitrarily small;

3. The sum of pulses of two electrons in a pair must be equal to zero $P_{\mathrm{ee}}=0$, i.e. electrons in the pair must have equal in magnitude and opposite in the direction pulses: $\mathbf{P}_{\mathrm{e} 1}=-\mathbf{P}_{\mathrm{e} 2}$.

Atomic electrons with equal quantum numbers $n, \ell$ and $m_{\ell}$, but and with antiparallel spins $s= \pm 1 / 2$, occupy different energy states in a strong magnetic field (Fig. 2b). For them, $\mathbf{P}_{\mathrm{e} 1} \neq-\mathbf{P}_{\mathrm{e} 2}$, so electrons cannot form a boson with $S=0$. Consequently, paired atomic electrons should be in equal energy states, which requires equal direction of their spins $\uparrow \uparrow, S=1$. Schematically, for a pair of atomic electrons the condition $\mathbf{P}_{\mathrm{e} 1}=-\mathbf{P}_{\mathrm{e} 2}$ can be realized if they are placed on the same line with atomic nucleus, opposite to each other (Fig. $\left.4, \mathbf{P}_{10}=-\mathbf{P}_{20}\right)$. In addition, such a location of electron pair allows them to compensate their mutual repulsion due to attraction to a positively charged nucleus.

The second condition - presence of attraction between paired electrons, is provided by the exchange interaction [9]. It is remarkable that the exchange interaction has the character of attraction in an atom for electrons with paralleldirected spins $\uparrow \uparrow$ only.

As it is known, the exchange interaction is associated with the indistinguishability of electrons (the principle of identity). It is characterized by the magnitude of exchange energy " $A$ ". The exchange energy represents an additional contribution to the total system energy.

It differs from zero only in case if wave functions of electrons overlap. The more the wave functions of electrons overlap, the greater is the exchange energy. In the atom, the energy of the Coulomb repulsion of electrons " $C$ " and the exchange energy " $A$ " are positive. (The exchange energy is negative for a hydrogen molecule). In contrast to the Coulomb electrostatic energy " $C$ ", the contribution of the exchange energy " $A$ " to the total system energy can have different signs depending on whether the spin part of wave function is symmetric or antisymmetric. The correction $\Delta E$ to the total system energy (5) connected with the interaction of electrons, is calculated in perturbation theory and is equal to: $\Delta E=C \pm A$,

where ' + ' refers to the antisymmetric $\uparrow \downarrow$ spin state $S=0$, and ' - ' refers to the symmetric $\uparrow \uparrow$ spin state $S=1$. Thus, thanks to the exchange interaction, two electrons with identically directed spins $\uparrow \uparrow$ are attracted together in an atom.

Since the exchange energy of electrons is comparable to the energy of their Coulomb repulsion, which is also compensated by the attraction to a positively charged atomic nucleus for paired electrons, it cannot be neglected in calculations. I.e., one can not consider the motion of one of the paired electrons in the field of the nucleus independent of the motion of the other electron. The motion of an electron is rigidly correlated with the motion of the second electron, as well as the probability of its finding at one or another point of space causes a point in space, at which the second electron is located with equal probability.

Electrons with identically directed spins $\uparrow \uparrow$, but with different quantum numbers $n_{\mathrm{b}}$ will be in different energy states. And the condition

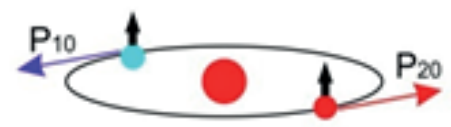

Fig. 4. Antiphase rotation of two electrons around a nucleus. 
$\mathbf{P}_{\mathrm{e} 1}=-\mathbf{P}_{\mathrm{e} 2}$ is not fulfilled for them. Therefore, the possibility for two electrons to have unidirectional spins at identical quantum numbers is associated with the need for additional antisymmetry in electron wave functions. Such an antisymmetry in the wave functions of the spatial motion of electrons can be ensured if, while on the same orbital, the paired electrons rotate around the nucleus $\left(\mathbf{P}_{10}=-\mathbf{P}_{20}\right.$, Fig. 4) and oscillate near the orbital $\left({ }^{1} \mathbf{P}_{1 \mathrm{~B}}=-{ }^{1} \mathbf{P}_{2 \mathrm{~B}}\right.$, Fig. $\left.5 a, b\right)$ in antiphase. In this case, the wave function of the spatial motion of the first electron will be an antisymmetric function relative to spatial motion of the second electron. The electron oscillations must occur both in the longitudinal and transverse magnetic field $\mathbf{B}$ directions $\left(\mathbf{P}_{1 \mathrm{~B}}=-\mathbf{P}_{2 \mathrm{~B}}\right.$, Fig. $\left.5 c, d\right)$. Such a motion allows two electrons in equal energy states to be in nonintersecting spatial regions. Fig. $5 b-d$ shows one and the same electron drawn on the orbital in the form of filled and empty circles is depicted conditionally. The Fig. 5b-d shows the case $n_{\mathrm{b}}=2$.

The projections of the oscillation pulse moments for each electron in a pair by the selected direction ( $Z$ axis) are not defined. However, these moments, just like the pulses themselves, are equal and opposite to each other in the direction:

$n_{1 \mathrm{~b}} \cdot \hbar=r_{1} \cdot \mathrm{P}_{1 \mathrm{~B}}=r_{2} \cdot\left(-\mathrm{P}_{2 \mathrm{~B}}\right)=-n_{2 \mathrm{~b}} \cdot \hbar$.

With an average $\left\langle r_{1}\right\rangle$ equal to the mean $\left\langle r_{2}\right\rangle$, this is equivalent to the equality of the modules, but with opposite signs, the quantum numbers of electron oscillations $n_{1 \mathrm{~b}}=-n_{2 \mathrm{~b}}$. Thus, the Pauli principle is true for two rigidly correlated electrons, which move in a fixed way with respect to each other in three-dimensional space around the nucleus.

It should be noted that, when a strong magnetic field orders the orbital moments of electrons, it "squeezes out" their orbital spatial uncertainty to other newly formed degrees of freedom, i.e. the oscillation moments of electrons. One may say that the amount of "chaos" is preserved in a closed system, if one applies efforts to its ordering.
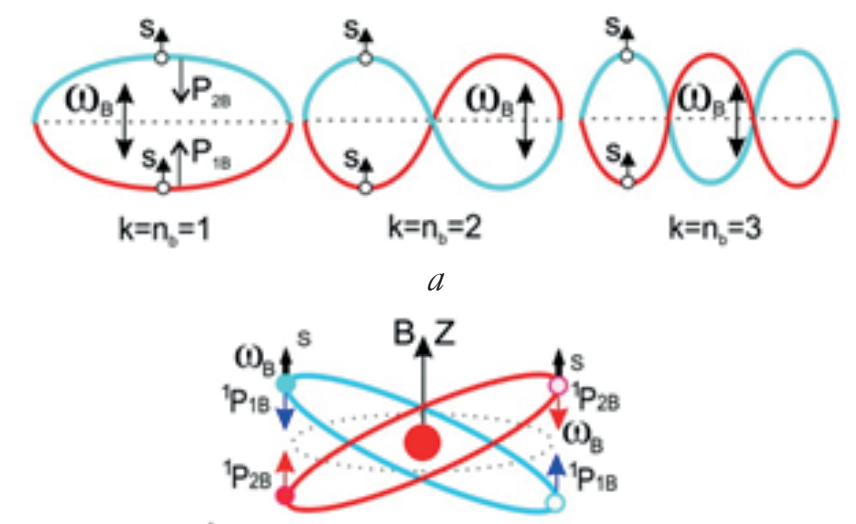

b.
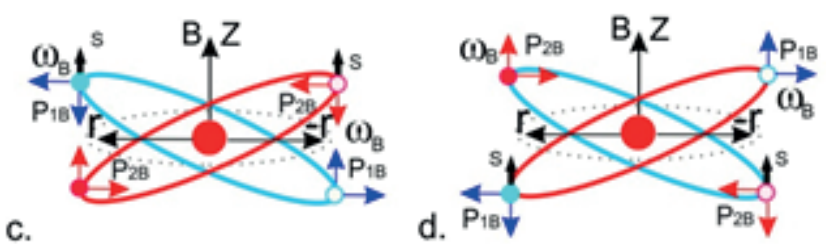

Fig. 5. $a, b$ - antiphase oscillations of paired electrons; $c, d$ antiphase oscillations of two paired electrons around nucleus close to orbital.

The total wave function of two electrons is equal to the product of symmetric spin wave function of two electrons $\Psi(S)$ by the wave function of their spatial motion $\Psi[1,2]$. It must be an antisymmetric function at rearrangement of electrons. If electrons change their places, Fig. $5 c$ is converted to Fig. $5 d$. Respectively, $\Psi\left[r_{1}, r_{2}\right]$ becomes $\Psi\left[r_{2}, r_{1}\right]$. The electron oscillation direction will change to opposite direction. Both wave functions $\Psi\left[r_{1}, r_{2}\right]$ and $\Psi\left[r_{2}, r_{1}\right]$ belong to the same energy eigenvalue. Therefore, the function: $\Psi(S) \cdot \Psi[1,2]=\Psi(S) \cdot\left\{\Psi\left[r_{1}, r_{2}\right]-\Psi\left[r_{2}, r_{1}\right]\right\}$ will become antisymmetric at electron rearrangement, but it will be symmetric with respect to the spatial reflection, if $r$ is replaced by $(-r): \Psi\left[-r_{1},-r_{2}\right]=\Psi\left[r_{1}, r_{2}\right]$ and $\Psi\left[-r_{2},-r_{1}\right]=\Psi\left[r_{2}, r_{1}\right]$ (Fig. $\left.5 c, d\right)$.

Thus, the pairing condition and the requirement of attraction between two electrons are automatically satisfied in a strong magnetic field due to the symmetric spin part of their wave functions $S=1$, and the requirement of antisymmetry of wave functions of the spatial motion of electrons with respect to each other automatically leads to zero sum of their moments $P_{\text {ee }}=0$. The fulfillment of these conditions and requirements is ensured by a rigid correlation of three-dimensional motion of two paired 
electrons in the central field of the nucleus in a strong magnetic field.

\section{INTRA-ATOMIC ELECTRON MAGNETIC RESONANCE}

The projections of magnetic moments on magnetic field direction do not change in a constant magnetic field. The direction of magnetic moments and spins can be changed by magnetic resonance methods. Known are methods of nuclear magnetic resonance (NMR) and electron paramagnetic resonance (EPMR). The essence of these methods consists in forced reorientation of magnetic moment of the nucleus (NMR) or of electron magnetic moment (EPMR) under the action of resonant electromagnetic high-frequency or microwave fields. The scheme of methods consists in placing the atoms of substance in a homogeneous magnetic field, where electron levels of an atom are split, and a resonant HF (microwave) magnetic field is applied perpendicular to the uniform magnetic field, which causes transitions between the split electron levels. In the NMR method, electron transitions occur between the levels of hyperfine atomic structure, and in the EPMR method, they occur between the levels of fine structure of an unpaired atomic electron.

The orbital magnetic moments $\mu_{\ell}$ with equal orbital moment $\ell$ precess around magnetic field B synchronously, with equal frequency $\omega_{\ell}=\mu_{\ell} \cdot B / \hbar, \mu_{\ell}=\ell \cdot \mu_{e}$ (Fig. 6a). The orbital magnetic moment $\mu_{\ell}$ creates an intrinsic magnetic field with a magnetic induction vector $\mathbf{B}_{\mu}$, which is calculated by the formula [10]:

$$
\mathbf{B}_{\mu}=\mu_{0} \frac{3 \mathbf{n}\left(\boldsymbol{\mu}_{\ell} \mathbf{n}\right)-\boldsymbol{\mu}_{\ell}}{r^{3}},
$$

where $\mu_{0}=1.26 \cdot 10^{-6} \mathrm{H} / \mathrm{m}$ is magnetic constant, $r$ is the distance from electron to the point at which the field is calculated, and $n$ is a unit vector in the $r$ direction. The magnetic induction vector $\mathbf{B}_{\mu}$ can be decomposed into two components: a magnetic field $\mathbf{B}_{\mu}^{\|}$directed parallel to the homogeneous field $\mathbf{B}$ and a magnetic field $\mathbf{B}_{\boldsymbol{\mu}}^{\perp}$ directed perpendicular to the field $\mathbf{B}$
(Fig. 6a). The vector by the magnetic induction $\mathbf{B}_{\mu}$ and its component $\mathbf{B}_{\mu}^{\perp}$ precess around $\mathbf{B}$ with a frequency $\omega_{\ell}=\mu_{\ell} \cdot B / \hbar, \mu_{\ell}=\mu_{e} \cdot \ell$. Once again, $\mathbf{B}_{\mu}^{\perp}$ precess perpendicular to B. Fig. $6 a$ shows two orbitals with equal orbital magnetic moments $\mu_{\ell}$ emanating from one point and lying in the same plane with $Z$ axis. If the last two states are permanently conserved, then perpendicular magnetic fields $\mathbf{B}_{\mu}^{\perp}$, created by two magnetic moments $\mu_{\ell}$, compensate each other. However, as rotational frequencies on the orbitals for electrons with different spins are not equal to each other: $\omega_{0}(s=1 / 2) \neq$ $\omega_{0}(s=-1 / 2)$, there are reasons to believe that orbital magnetic moments $\mu_{\ell}$ create magnetic fields, which perpendicular components $\mathbf{B}_{\mu}^{\perp}$ are not compensated. In addition, magnetic fields

$\mathbf{B}_{\mu}^{\perp}$ are not compensated for the odd elements. The magnetic fields $\mathbf{B}_{\mu}^{\|}$remain uncompensated until the whole subshell $\ell$ is filled.

The interaction energy of magnetic field $\mathbf{B}$ with orbital magnetic moment of the electron is $\Delta E_{\ell}=-\ell \cdot \mu_{\mathrm{e}} B$, with the spin magnetic moment of the electron $\Delta E_{\mathrm{s}}=-2 \mu_{\mathrm{e}} \cdot s \cdot B$. The energy between levels for a pair of electrons $m_{\mathrm{S}}= \pm 1 / 2$ is equal to $\Delta E m_{\mathrm{S}}=2 \mu_{\mathrm{e}} \cdot B$. For the $p$-subshell $(\ell=1)$, the energy $\Delta E m_{\ell}$ between neighboring levels $m_{\ell}$ is half the energy $\Delta E m_{\mathrm{S}}$ (Fig. 6b). For the $d$-subshell $(\ell=2)$, the energy $\Delta E m_{\ell}$ is equal to the energy $\Delta E m_{S}$ (Fig. $6 c$ ). Therefore, for the $d$-subshell, the rotation frequency $\omega_{\ell}=2 \cdot \mu_{\mathrm{e}} \mathrm{B} / \mathrm{h}$ of magnetic fields $\mathbf{B}_{\mu}$ coincides with transition frequency $\omega_{\mathrm{s}}=\Delta E m_{\mathrm{S}} / \hbar=2 \cdot \mu_{\mathrm{e}} B / \hbar$ between the spin levels $m_{\mathrm{S}}=1 / 2$ and $m_{\mathrm{S}}=-1 / 2$. This enables to appear transitions between spin levels $m_{\mathrm{s}}=1 / 2$ and $m_{\mathrm{S}}=-1 / 2$ stimulated by rotating magnetic fields $\mathbf{B}_{\boldsymbol{\mu}}^{\perp}$. In case of a $p$-subshell, the rotation of two oppositely directed magnetic fields $\mathbf{B}_{\boldsymbol{\mu}}^{\perp}$ with a frequency $\omega_{\ell}$ can be represented as a sum of fields rotating with multiple $\omega_{\ell}$, including frequency $2 \omega_{\ell}$. Therefore, transitions between levels $m_{\mathrm{S}}=1 / 2$ and $m_{\mathrm{S}}=-1 / 2$ stimulated by fields $\mathbf{B}_{\mu}$ are also possible for the $p$-subshell. 

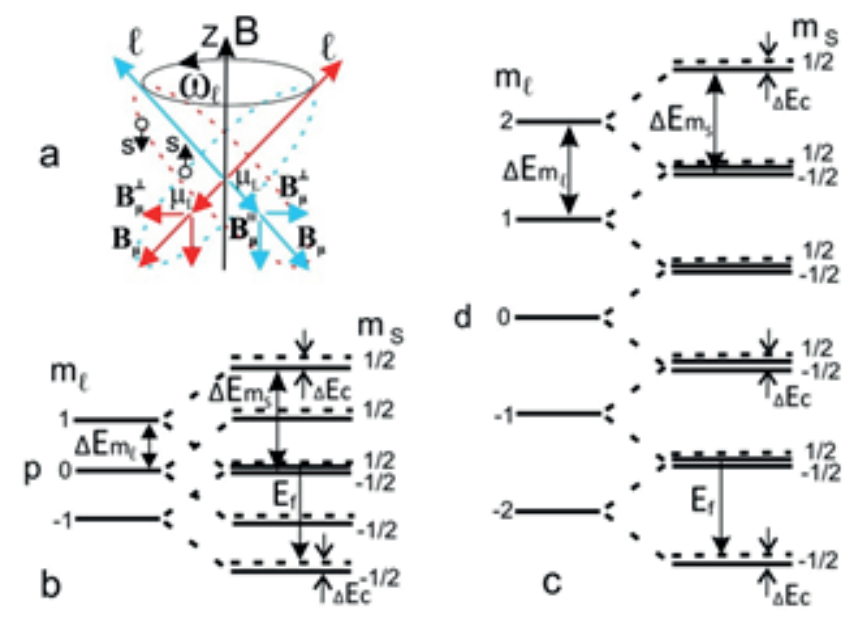

Fig. 6. $a-\omega_{\ell}$ frequency precession of electron orbital magnetic moments $\mu_{\ell}$ and magnetic fields $\mathbf{B}_{\mu}$ and $\mathbf{B}_{\mu}^{\perp}$ generated by them; $b, c$-splitting of $p$ - and $d$-subshells in a strong magnetic field.

Rotating magnetic fields $\mathbf{B}_{\mu}^{\perp}$ will stimulate transitions with energy release $E_{\mathrm{f}}$ (Fig. 6b,c), i.e. transitions from levels $m_{\mathrm{S}}=1 / 2$ to levels $m_{\mathrm{s}}=-1 / 2$. Inverse transitions are forbidden, because they proceed with energy absorption that must be taken from somewhere, and an external generator of rotating magnetic fields $\mathbf{B}_{\mu}$ is absent. The transition from level $m_{\mathrm{S}}=1 / 2$ will occur to intermediate transport level, from which the electron pair will move to orthoboson state. Because of increased Coulomb repulsion between electrons, transport levels will be above spin levels both for $m_{\mathrm{S}}=1 / 2$ and $m_{\mathrm{S}}$ $=-1 / 2$ by the value $\Delta E_{c}$ (Fig. 6 ). The energy $\Delta E m_{\mathrm{S}}$ between levels $m_{\mathrm{S}}= \pm 1 / 2$ should be much higher than $\Delta E_{c}: \Delta E m_{S}>\Delta E_{c}$ (Fig. 6b,c). The transport levels in Fig. 6b,c are shown as dashed line. The energy released during the transition is $E_{\mathrm{f}}=\Delta E m_{\mathrm{S}}-\Delta E_{\mathrm{c}}$.

Consequently, in a strong magnetic field $\mathbf{B}$, precessing orbital magnetic moments $\mu_{\ell}$ create internal, rotating magnetic fields $\mathbf{B}_{\mu}$ that stimulate forced transitions between atomic levels $m_{S}=1 / 2$ $\rightarrow m_{\mathrm{S}}=-1 / 2$. Thus, in a strong magnetic field, the spin-orbit interaction of internal electrons, leads, due to intra-atomic electron magnetic resonance (IAEMR), to pairing of two atomic electrons into an ortho-boson.

\section{ATOM AS Z/2 ACTIVE MEDIUM}

The energy between the spin levels $m_{\mathrm{S}}=1 / 2$ и $m_{\mathrm{S}}=-1 / 2$ will be, in a strong magnetic field, approximately the same for all atoms, regardless of their electron shell and charge of the $Z$ nucleus, and will be equal to $\Delta E m_{\mathrm{S}}=2 \mu_{\mathrm{e}} \cdot B$ (with exactness up to newly arising internal magnetic fields $\mathbf{B}_{\mu}$ ). Moreover, the atom has $Z / 2$ such two-level systems. I.e., each atom in a strong magnetic field represents a $Z / 2$ two level active medium.

As already mentioned, thanks to the IAEMR, an interlevel transition is performed with the emission of a photon with energy $E_{\mathrm{f}} \approx 2 \mu_{\mathrm{e}} \cdot B$. The proton energy amounts, with an external magnetic field in the range of $10 \div 100 \mathrm{~T}$, to $10^{-3} \div 10^{-2} \mathrm{eV}$, which corresponds to a frequency of $0.3-3 \mathrm{THz}$ or a wavelength of $1-0.1 \mathrm{~mm}$. The emitted photon $E_{\mathrm{f}}$, propagating in the active medium, stimulates the forced transitions with the same energy both inside the atom in which it was generated (Fig. 7a) and in other surrounding atoms. Thus, the $Z / 2$ active medium will produce coherent radiation in the terahertz range. Transitions from the lower $m_{\mathrm{S}}=-1 / 2$ level to the upper $m_{\mathrm{S}}=1 / 2$ under the action of the photon $E_{f}$ will not occur, since the transition energy $E_{i}$ is greater than $E_{f}$ (Fig. $7 a$ ). Inverse transitions are unlikely, because more rapid transitions to ortho-boson states and transitions of ortho-bosons from high-lying levels to low-lying levels will occur at once (Fig. 7b). In the end, ortho-bosons will occupy the lowest-lying level. Transitions to low-lying levels will be carried out by electron ortho-bosons, probably, with the emission of two photons. Since transitions occur between internal electron levels of the atom, the energies of these photons depend on the charge of nucleus $Z$ and lie in the range from hard ultraviolet to hard $X$-rays of $10^{2}-10^{5} \mathrm{eV}$ [11]. The energies of these transitions are characteristic for each $Z$. Thus, coherent radiation will be produced at other wavelengths (Fig. 7b), along with terahertz radiation. Apparently, exactly that radiation in the $X$-ray range was discovered by 
A.V. Karabut [12-13] in experiments with a highcurrent glow discharge and by S.V.Adamenko in experiments with the irradiation of metals by a powerful electron beam [14]. Moreover, Karabut A.V. observed intensive generation of $X$-ray radiation in the form of narrowly directed beams in his experiments [13]. The diameter of the microbeam at a distance of $200 \mathrm{~mm}$ from the cathode was estimated to be 10-20 $\mu \mathrm{m}$, and the angular divergence to be $\sim 10^{-4}$.

Electrons in ortho-bosons rotate around the nucleus and oscillate near the lowest-lying orbital in antiphase with frequency $\omega_{0}\left(\ell=0, n_{\mathrm{b}}=1\right)$. The phases of neighboring electrons are shifted by $360^{\circ} / P_{\mathrm{e}}$, where $P_{\mathrm{e}}$ is the number of paired electrons in the shell. The trajectories of two moving paired electrons represent closed spirals, which are enclosed into each other and similar to the spiral of DNA molecules. The trajectories of several ortho-bosons are located on the surface of toroid and form several spirals resembling the DNA code of the main character of the film "The Fifth Element" - Lila [15]. Such a comparison is connected with the phenomenon of transmutation (transformation) of certain chemical elements into other chemical elements in microbiological systems [16-18]. Apparently, depending on the values of the magnetic field $\mathbf{B}$, the charge of nucleus $Z$ and the number of

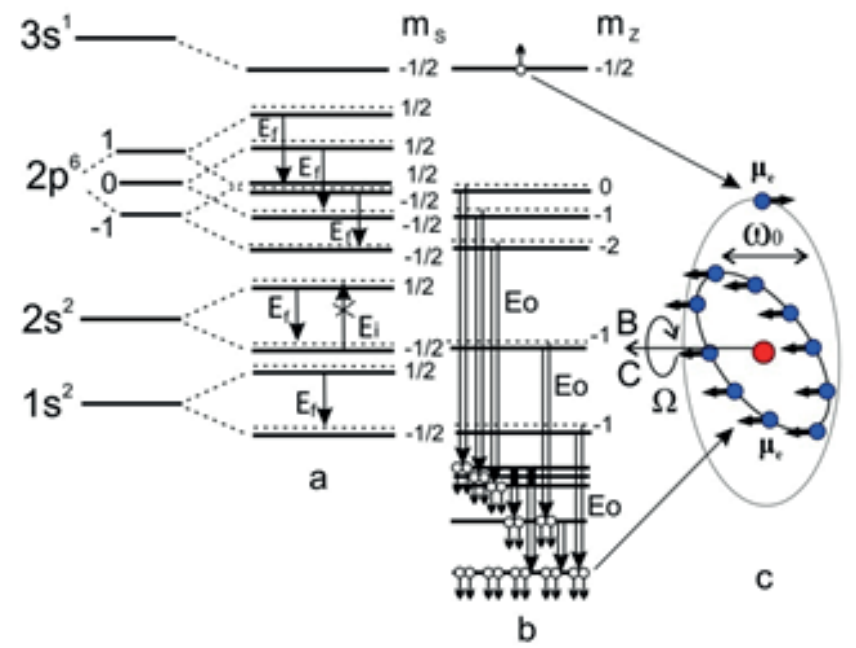

Fig. 7. Atom as $Z / 2$ active medium. a-sodium atom radiation of forced photons $E_{\rho}$ in terahertz range; $b$ - sodium radiation realized by electron ortho-bosons; $c$ - sodium transatom. paired electrons $P_{\mathrm{e}}$, the toroid may have a circle, an elongated or flattened ellipse in its section.

As experiments [18-20] have shown, chemical $d$-elements can act as catalysts to increase the efficiency of formation of ortho-bosons in a $\mathrm{Z} / 2$ active medium. This is due to the fact that rotation frequency $\omega_{\ell}$ of magnetic fields $\mathbf{B}_{\mu}$ almost coincides, for the $d$-subshell, with the frequency $\omega_{\mathrm{s}}$ of transition between spin levels $m_{\mathrm{S}}=1 / 2$ and $m_{\mathrm{S}}=-1 / 2$. The $d$-elements include: ${ }_{21} \mathrm{Sc}_{30} \mathrm{Zn},{ }_{39} \mathrm{Y}_{48} \mathrm{Cd},{ }_{57} \mathrm{La},{ }_{72} \mathrm{Hf}-{ }_{80} \mathrm{Hg},{ }_{89} \mathrm{Ac},{ }_{104} \mathrm{Rf}^{-}{ }_{112} \mathrm{Cn}$. By chance or not, scientists engaged in the research of cold nuclear synthesis will extract Ti, Ni, Zr, $\mathrm{Pd}$, and $\mathrm{Au}$ out of these elements.

Thus, it is possible to create sources of coherent radiation in the terahertz range and radiation ranges: from hard ultraviolet to hard $X$-ray $\sim 10^{2}-10^{5} \mathrm{eV}$.

\section{FORMATION OF TRANSATOMS AND TRANSMOLECULES}

A spin electron Bose condensate will form in the atom at lowest-lying levels. An atom possessing such Bose condensate is called the Transatom. Fig. $7 c$ shows schematically a sodium transatom. Magnetic induction vector of sodium transatom created by electron spin magnetic moments at nucleus location has a value of $\sim 10^{6} \mathrm{~T}$. The transatom due to interaction of its summarized magnetic moment $\mu_{\mathrm{e}} \cdot P_{\mathrm{e}}$ with external magnetic field $\mathbf{B}$, will precess $\Omega=\mu_{\mathrm{e}} P_{\mathrm{e}} B / \hbar$ (Fig. $7 c$ ) around direction $\mathbf{B}$.

All electron spin magnetic moments in Bose condensate are directed in one direction. These moments create a giant inhomogeneous and anisotropic magnetic field $\sim 10^{6}-10^{9} \mathrm{~T}$ in the entire region of atom, including in the region of nucleus location (6) [11]. This field forms an electromagnetic trap in the first electron boson orbital with a radius $R_{\mathrm{Z}}$ with magnetic field irregularity of $10^{2}-10^{6} \mathrm{~T}$ on the nucleus diameter. Fig. 8 demonstrates, in relative units, the dependence of magnetic induction vector $\mathbf{B}_{\mathrm{S}}$ from the distance to nucleus $C\left(R_{\mathrm{Z}}\right)$ along the $C$ axis (Fig. $7 c)$. The distance $C\left(R_{z}\right)$ is normalized 
for $R_{z}=1$. Negative values of magnetic induction vector $\mathbf{B}_{\mathrm{S}}$ mean that magnetic field in the central region of transatom is directed in the direction opposite to electron magnetic moment direction. When one or several nuclei move within a magnetic trap, an inhomogeneous and anisotropic magnetic field causes changes in the energy levels of protons and neutrons. As a result, nuclear structure and their properties change. The nuclei are transformed into Transnuclei. Transatoms with transnuclei represent a new state of matter: spin-nuclideelectron condensate [11].

The transatom has a magnetic feature. Transatoms are attracted to each other. When two transatoms converge, their electronic shells are combined into one shell, common for both nuclei. This results in the creation of a binuclear system. Other transatoms can join that system subsequently. Thus, a multinuclear system - nuclear molecule - transmolecule is formed. The transmolecule is located in the region of inhomogeneous and anisotropic magnetic field. Consequently, the space, in which transnuclei move in the transmolecule, is inhomogeneous and anisotropic. Thus, motion integrals are not conserved in transnuclear interaction: momentum conservation law, angular momentum (spin) conservation law, and, apparently, energy conservation law are violated. As a result, nonradiative, low-energy multinuclear reactions automatically take place in nuclear transmolecule [11-14, 16-27].

Not all atomic electrons, but only a part of them can, in the process of forced transitions, be transformed into ortho-bosons. Thus, each atomic nucleus $Z$ can have $Z / 2$ "chemical" transelements or "transatomic chimeras". A part of "transatomic chimera" will be represented by electrons in a paired boson state, the other part will be represented by electrons, which fill the "chimera" orbitals. Here, "chimera" orbitals should be understood as "traditional" orbitals with corrections due to magnetic and electric field influence created by transatomic paired

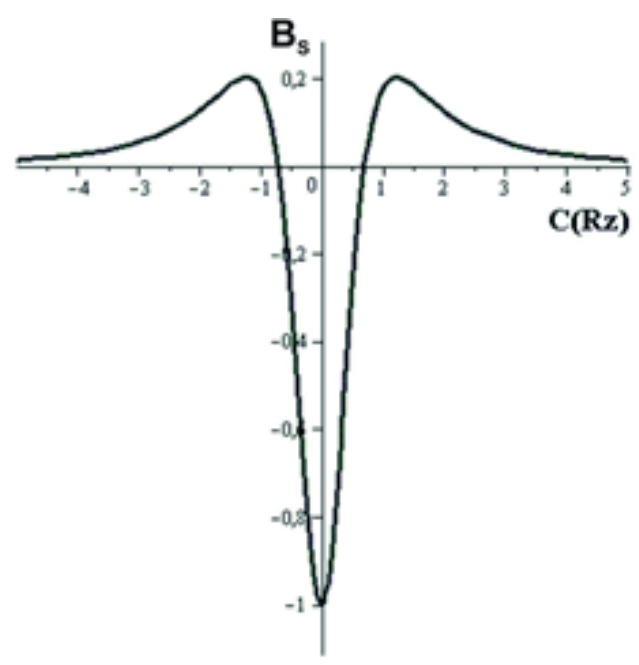

Fig. 8. Dependence of magnetic induction vector value $\mathbf{B}_{S}$ in relative units along axis $C$.

electrons of the transatom on its unpaired electrons. Thus, many other transelements are added to the existing chemical elements in D.I. Mendeleev Table. So, if one confines himself to nuclei with $Z$ charge from 2 to 100 , then the number of transelements will be $\sim 2500$.

Thus, the transatoms generated by strong magnetic fields of 20-100 $T$ have ultrastrong magnetic fields of $10^{6}-10^{9} \mathrm{~T}$ in their volume and at nucleus location [11]. In a strong magnetic field, atoms inevitably turn into transatoms, which inevitably enter into low-energy nuclear reactions. The proposed hypothetical mechanism for the conversion of atoms into transatoms requires experimental verification.

\section{CONCLUSION}

In a strong magnetic field $\mathbf{B}$, the uncertainty of the $x$-, $y$ - and $z$-components of the orbital moments of internal electrons is removed. Orbital moments of all electrons are "frozen-in" in a magnetic field. For this purpose, the value of field $\mathbf{B}$ should be comparable with magnetic fields generated by electron orbital moments $\mathbf{B}_{\mu}$, i.e. more than $20 \mathrm{~T}$.

Thanks to Coulomb forces, the motion of electrons in atom acquires a new degree of freedom, i.e. oscillations. Oscillations can be quantized by introducing a new quantum number. 
The orbital moments of electrons precessing around the field $\mathbf{B}$ generate theirown, intra-atomic magnetic fields $\mathbf{B}_{\mu}$, which also precess around the field $\mathbf{B}$. The components $\mathbf{B}_{\mu}\left(\mathbf{B}_{\mu}^{\perp}\right)$ orthogonal to the field $\mathbf{B}$ stimulate forced transitions between electronic levels differing only in the direction of electron spins $m_{\mathrm{S}}=1 / 2 \rightarrow m_{\mathrm{S}}=-1 / 2$. Such transitions result into formation of ortho-bosons with $S=1$. The possibility of existence of orthobosons is provided by exchange interaction between electrons and a rigid correlation in the quantum, three-dimensional motion of paired electrons around the nucleus. Orthobosons on the orbitals closest to nucleus create a spin electron condensate. The atomic structure changes. There is a complete reorganization of electron configurations of all atomic electrons. Atoms in a strong magnetic field inevitably turn into transatoms.

A strong magnetic field $\mathbf{B}$ that identically interacts with all electrons of all atoms generates a $Z / 2$ active medium. The $Z / 2$ deexcitation of active medium is accompanied by intense coherent radiation lying in terahertz range and ultraviolet to $X$-ray ranges.

Spin electron condensate creates a giant inhomogeneous anisotropic magnetic field $\mathbf{B}_{\mathrm{S}}$ in the transatom volume. This field changes the structure of a nucleus, turning it into a transnucleus. In addition, the $\mathbf{B}_{\mathrm{s}}$ field changes interaction way of transnuclei, both with each other and with surrounding Bose electrons. A transnuclear transatom is a new state of matter: spin-nuclide-electronic condensate (SNEC).

The SNEC is a changed state of atomic and nuclear structure of chemical elements. The physical and chemical properties of elements change in this state. Transatoms are attracted to each other and form transmolecules with new properties that differ from those of ordinary molecules.

The SNEC transnuclei can enter into nuclear reactions. A peculiarity of these nuclear reactions is the absence of irradiation during their implementation and the absence of radioactive isotopes in their products. Nuclear reactions occur after the formation of transmolecules automatically. Consequently, cross section values of nuclear reactions are comparable with cross sections values of atomic processes.

Spin-nuclide-electronic condensate posesses physical properties unknown up to present time. It is easy and, at the same time, difficult to predict what opportunities can be realized using these properties in science and technology. The transatom is inexhaustible...

\section{REFERENCES}

1. Ul'yanov VI (Lenin). Materializm $i$ empiriokrititsizm. Polnoe sobr. soch., 18(5):231. Moscow, Politicheskaya literatura Publ., 1968.

2. Kadomtsev BB. Tyazhely atom v sverkhsil'nom magnitnom pole [Heavy atom in an ultrastrong magnetic field]. ZhETF, 1970, 58(5):1765-1769 (in Russ.).

3. Kadomtsev BB, Kudryavtsev VS. Veshchestvo $\mathrm{v}$ sverkhsil'nom magnitnom pole [Matter in an ultrastrong magnetic field]. ZhETF, 1972, 62(1):144-152 (in Russ.).

4. Slichter ChP. Principles of magnetic resonance. New York, Harper \& Row Publishers, 1963, 246 p.

5. Fock VA. Bemerkung zur Quantelung des harmonischen Oszillators im Magnetfeld. Z.Phys., 1928, 47:446-448.

6. Heiss WD, Nazmitdinov RG. Orbital magnetism in small quantum dots with closed shells. Pis'ma v ZhETF, 1998, 68(12):870-875.

7. Matveev AN. Atomic physics. Moscow, Vysshaya shkola Publ., 1989, 439 p.

8. Cooper LN. Bound electron pairs in a degenerate Fermi gas. Phys.Rev, 1956, 104:1189.

9. Heisenberg W. Über die Spektra von Atomsystemen mit zwei Elektronen. Z. Phys., 1926, 39(7):499-518.

10. Landau LD, Lifshits YeM. Teoriya polya [Field theory]. Moscow, Nauka Publ., 1973, 512 p.

11. Mishinsky GV. Magnitnye polya transatomov. Spinovy-nuklidny-elektronny kondensat 
[Magnetic fields of transatoms. Spin-nuclideelectronic condensate]. International Journal of Unconventional Science, 2017, 15-16(5):6-25 (in Russ.).

12. Karabut AB. Analiz rezultatov registratsii izbytochnoy teplovoy moshchnosti, primesnykh nuklidov $\mathrm{s}$ izmenennym sootnosheniem izotopov i pronikayushchego izlucheniya $\mathrm{v}$ eksperimentakh s silnotochnym tleyushchim razryadom [Analysis of the results of recording excess heat power, impurity nuclides with a modified natural ratio of isotopes and penetrating radiation in experiments with a high-current glow discharge]. Proc. of 7 th Russian Conference on Cold Transmutation of Nuclei of Chemical Elements (RCCTNCE), Moscow, 2000, p. 27 35 (in Russ.).

13. Karabut AB. Zavisimost' proizvodstva izbytochnoy teplovoy moshchnosti, produktov yadernykh reaktsiy i emissii rentgenovskogo izlucheniya ot eksperimentalnykh parametrov silnotochnogo tleyushchego razryada Dependence of the production of excessive thermal power, products of nuclear reactions and emission of $\mathrm{X}$-ray radiation on the experimental parameters of a high-current glow discharge. Proc. of 9th Russian Conference on Cold Transmutation of Nuclei of Chemical Elements, Moscow, 2002, p. 86-98 (in Russ.).

14. Adamenko SV, Selleri F, A. van der Merwe. Controlled Nucleosynthesis. Breakthroughs in Experiment and Theory. Series: Fundamental theories in Physics, Springer, 2007, 156, 780 p., http://www.proton21.com.ua/articles/ Booklet_ru.pdf.

15. Besson L, Kamen RM. The Fifth Element, film, 1997.

16. Vysotskii VI, Kornilova AA. Nuclear transmutation of stable and radioactive isotopes in biological systems. India, Delhi, Pentagon Press, 2010.

17. Kornilova AA. Vysotskii VI. Sintez i transmutatsiya stabil'nykh i radioaktivnykh izotopov $\mathrm{v}$ biologicheskikh sistemakh [Synthesis and transmutation of stable and radioactive isotopes in biological systems]. Radioelektronika. Nanosistemy. Informatsionbye Tekbnologii (RENSIT), 2017, 9(1):52-64 (in Russ.).

18. Kurashov VM, Sakhno TV. Mikrobiologicheskiy sposob transmutatsii khimicheskikh elementov i prevrashcheniya izotopov khimicheskikh elementov [Microbiological method of transmutation of chemical elements and conversion of isotopes of chemical elements]. Patent RU 2 563511 C2 May 15, 2014.

19. Simakin AV, Shafeev GA. Vliyanie lazernogo oblucheniya nanochastits V vodnykh rastvorakh soli urana na aktivnost' nuklidov [The influence of laser irradiation of nanoparticles in aqueous solutions of uranium salts on the activity of the nuclides]. Kvantovaya elektronika, 2011, 41(7):614-618 (in Russ.).

20. Andreev SN, Shafeev GA. Nelineynoe tushenie radioaktivnosti vodnykh rastvorov soley nuklidov pri lazernoy ablyatsii nanochastits metallov [Onlinear quenching of the radioactivity of aqueous solutions of salts of nuclides by laser ablation of nanoparticles of metals]. RENSIT, 2017, 9(1):65-73 (in Russ.).

21. Mishinsky GV. Transatomy-transyadra i ikh svoystva [Transatoms - Transnuclei, and their properties. Proceedings of 18 th Russian conference on cold transmutation of nuclei of chemical elements, 2012, Moscow, pp. 94-106 (in Russ.).

22. Mishinsky GV. Mnogoyadernye reaktzii $\mathrm{v}$ kondensirovannom gelii [Multinuclear reactions in condensed helium]. RENSIT, 2017, 9(1):94-105 (in Russ.).

23. Proceedings of 1-23th Russian Conference on Cold Transmutation of Nuclei of Chemical Elements (in Russ.).

24. Proceedings of the 1-19th International Conferences on ColdFusion (CondensedMatter Nuclear Science). 
25. Balakirev VF. Krymskiy VV, Bolotov BV. et al. V zaimoprevrashcheniye khimicheskikh elementov [nterconversion of chemical elements]. Ed. Balakirev VF. Ekaterinburg, UrO RAN Publ., 2003, 64 p.

26. Kuznetsov VD, Mishinsky GV, Penkov FM, Arbuzov VI, Zhemenik VI. Low energy transmutation of atomic nuclei of chemical elements. Annales de la Fondation Louis de Broglie, 2003, 28(2):173-214.

27. Mishinsky GV, Kuznetsov VD. Element distribution in the products of low energy transmutation. Nucleosynthesis. Annales de la Fondation Louis de Broglie, 2008, 33(3-4):331356; Proceedings of 14-th Russian Conf. on cold transmutation of nuclei of chemical elements, 2008, Moscow, pp. 79-97 (in Russ.). 